\title{
LIXIVIAÇÃO DE ÍONS EM COLUNAS DE SOLO DEFORMADO E INDEFORMADO
}

\author{
SILVIO C. SAMPAIO ${ }^{1}$, FRANCIELLE A. CAOVILLA ${ }^{2}$, MIGUEL A. U. OPAZO ${ }^{3}$, \\ LÚCIA H. P. NÓBREGA ${ }^{4}$, MORGANA SUSZEK ${ }^{5}$, ADRIANA SMANHOTTO ${ }^{6}$
}

RESUMO: Neste trabalho, foi avaliada a dinâmica da lixiviação de íons em colunas de solo deformado e indeformado. $\mathrm{O}$ solo utilizado era proveniente de área fertirrigada com água residuária da suinocultura diluída em $0 \%, 25 \%, 50 \%, 75 \%$ e cultivada com soja. Foram construídas curvas de eluição para nitrato, potássio e condutividade elétrica. De acordo com os resultados obtidos nas colunas de solo deformado, e proporcionalmente aos tratamentos, o nitrato apresentou maior mobilidade, seguido pela concentração de sais totais e do potássio. A metodologia de colunas de solo indeformado não se mostrou adequada, devido, possivelmente, à compactação do solo durante a amostragem e à dificuldade no bloqueio de caminhos preferenciais ao longo de toda a coluna.

PALAVRAS-CHAVE: água residuária, meio ambiente, dinâmica de íons.

\section{IONS LEACHING IN DEFORMED AND UNDEFORMED SOIL COLUMNS}

\begin{abstract}
In this work it was evaluated the dynamics of ions leaching in deformed and undeformed soil columns. The used soil proceeded from an area irrigated with swine wastewater in different dilutions $(0 \%, 25 \%, 50 \%$ and $75 \%)$ and cultivated with soybean. Breakthrough curves for nitrate, potassium and electric conductivity were constructed. According to the results, in the deformed and undeformed soil columns, the nitrate presented greater mobility, followed by the total salts and the potassium, proportional to the treatments. The methodology of undeformed soil columns was not adequate, possibly due to the compacting during the sampling and the difficulty in the blockade of preferential ways throughout all columns.
\end{abstract}

KEYWORDS: wastewater, environmental, ions dynamics.

\section{INTRODUÇÃO}

A suinocultura representa para o Brasil uma importante atividade econômica, sendo o Estado do Paraná responsável pelo abate de cerca de 5,1 milhões de cabeças de suínos por ano (EMBRAPA, 2006). Entretanto, a preocupação com a poluição ambiental é uma das maiores ameaças à sobrevivência e à expansão da suinocultura nos grandes centros produtores, devido à geração de elevada quantidade de dejetos.

Por se tratar de técnica de disposição que reduz os custos de tratamento e traz benefícios à agricultura, o aproveitamento de águas residuárias da suinocultura na fertirrigação das culturas tem despertado o interesse dos agricultores. Porém, essa água, quando aplicada acima da capacidade de suporte do solo, pode provocar contaminação do solo e das águas subterrâneas através da lixiviação de nutrientes (PELISSARI et al., 2009; ANAMI et al., 2008; DAL BOSCO et al., 2008; ANAMI et

\footnotetext{
${ }^{1}$ Pesquisador em Recursos Hídricos e Saneamento Ambiental. UNIOESTE/CASCAVEL/CCET/PGEAGRI/RHESA, Cascavel - PR, Fone: (0XX45) 3220.3262, ssampaio@ unioeste.br.

${ }^{2}$ Doutoranda em Recursos Hídricos e Saneamento Ambiental, UNIOESTE/CASCAVEL/CCET/PGEAGRI/RHESA, Cascavel - PR, Fone: (0XX45) 3220.3262, francaovilla@yahoo.com.br.

${ }^{3}$ Pesquisador em Engenharia de Sistemas Agroindustriais, UNIOESTE/CASCAVEL/CCET/PGEAGRI/ESA, Cascavel - PR, Fone: (0XX45) 3220.3228, mopazo@ unioeste.br.

${ }^{4}$ Pesquisadora em Engenharia de Sistemas Agroindustriais. UNIOESTE/CASCAVEL/CCET/PGEAGRI/ESA, Cascavel - PR, Fone: (0XX45) 3220.3195, lhpn@unioeste.br.

${ }^{5}$ Doutoranda em Recursos Hídricos e Saneamento Ambiental, UNIOESTE/CASCAVEL/CCET/PGEAGRI/RHESA, Cascavel - PR, Fone: (0XX45) 3220.3262, morgana@utfpr.edu.br.

${ }^{6}$ Pesquisadora em Recursos Hídricos e Saneamento Ambiental, PUC, Toledo - PR, Fone: (0XX45) 3277.8600, adrianasmanhotto@hotmail.com.

Recebido pelo Conselho Editorial em: 5-6-2008

Aprovado pelo Conselho Editorial em: 12-12-2009
} 
al., 2007; BAUMGARNTER et al., 2007; SAMPAIO et al., 2007; FRIGO et al., 2006; CAOVILLA et al., 2005; SUSZEK et al., 2005; GOMES et al., 2004).

Para COSTA et al. (1999), os íons disponibilizados na solução do solo podem ser adsorvidos ao solo, absorvidos pelas plantas ou lixiviados das camadas superficiais do solo. As perdas de nutrientes causadas pelo fenômeno da lixiviação são importantes pelo fato de significar baixas eficiências de utilização de nutrientes pelas culturas e, por consequência, menores rendimentos. Quando lixiviados, os nutrientes incorporam-se ao lençol freático pelo processo de drenagem interna e, assim, são transportados a grandes distâncias, contaminando o lençol freático e também os mananciais de água potável (KONRAD, 2002).

Segundo SANTOS et al. (2002), a lixiviação de íons varia com os atributos físicos do solo, como textura, estrutura, profundidade do perfil e, principalmente, porosidade. Entre os atributos químicos que afetam a lixiviação, estão a capacidade de retenção de íons e o pH.

Neste contexto, o presente trabalho teve por objetivo avaliar a dinâmica de lixiviação de nitrato, potássio e sais (a partir da condutividade elétrica) em colunas de solo deformado e indeformado, de um Latossolo Vermelho distroférrico típico, provindo de área cultivada com soja e submetida à fertirrigação com água residuária da suinocultura.

\section{MATERIAL E MÉTODOS}

A semeadura da soja foi realizada em ambiente protegido em Latossolo Vermelho distroférrico típico (EMBRAPA, 1999). Realizou-se a fertirrigação da cultura, via gotejamento, com água residuária da suinocultura (ARS), durante 60 dias, com lâmina média de 4,28 mm dia ${ }^{-1}$, seguindo metodologia de LONGO et al. (2006). Os tratamentos foram considerados de acordo com a concentração de ARS na água de irrigação, em níveis de $0 ; 25 ; 50$ e 75\%, correspondendo aos tratamentos T1, T2, T3 e T4, respectivamente. Os resultados dos parâmetros físico-químicos determinados na água utilizada em cada tratamento são apresentados na Tabela 1.

TABELA 1. Características físico-químicas das águas de irrigação. Physical-chemical characteristics of water for irrigation.

\begin{tabular}{lccrrr}
\hline \multirow{2}{*}{ Parâmetro } & \multirow{2}{*}{ Unidade } & \multicolumn{4}{c}{ Tratamento } \\
\cline { 3 - 6 } & & $\mathrm{T} 1$ & $\mathrm{~T} 2$ & $\mathrm{~T} 3$ & \multicolumn{1}{c}{$\mathrm{T} 4$} \\
\hline $\mathrm{pH}\left(\mathrm{CaCl}_{2}\right)$ & - & 7,69 & 6,78 & 6,96 & 6,81 \\
Condutividade elétrica & $\left(\mu \mathrm{S} \mathrm{cm}^{-1}\right)$ & 0,11 & 5,44 & 8,39 & 10,82 \\
Alcalinidade & $\mathrm{g} \mathrm{CaCO}_{3} \mathrm{~L}^{-1}$ & 0,09 & 1,38 & 2,20 & 3,28 \\
Turbidez & $\mathrm{NTU}^{-1}$ & 0,86 & 500,00 & 670,00 & $1.135,00$ \\
Sólidos totais & $\mathrm{g} \mathrm{L}^{-1}$ & - & 1,96 & 4,26 & 5,14 \\
Sólidos voláteis & $\mathrm{g} \mathrm{L}^{-1}$ & & 1,76 & 2,04 & 3,40 \\
Fósforo (P) & $\mathrm{mg} \mathrm{L}^{-1}$ & 81,07 & 431,06 & 944,54 & $1.009,40$ \\
Nitrogênio amoniacal & $\mathrm{mg} \mathrm{L}^{-1}$ & - & 520,00 & 790,00 & 900,00 \\
Nitrato & $\mathrm{mg} \mathrm{L}^{-1}$ & - & 28,00 & 42,00 & 70,00 \\
Nitrogênio total & $\mathrm{mg} \mathrm{L}^{-1}$ & - & 530,00 & 710,00 & $1.150,00$ \\
Potássio $(\mathrm{K})$ & $\mathrm{mg} \mathrm{L}^{-1}$ & - & 143,00 & 190,00 & 220,00 \\
\hline
\end{tabular}

Após a colheita da cultura, para cada tratamento, retiraram-se amostras de solo indeformado e deformado. As colunas de solo indeformado foram amostradas conforme BREDA (2003), a partir de um penetrômetro de placa de aço acoplado a um trator, o qual manteve velocidade de penetração de $0,005 \mathrm{~m} \mathrm{~s}^{-1}$; foi utilizada parafina fundida para o preenchimento entre as paredes da coluna e o solo, com o intuito de bloquear possíveis caminhos preferenciais.

Para a montagem das colunas de solo deformado, coletaram-se amostras com o auxílio de um trado, tendo como referência as camadas de $0-0,20 \mathrm{~m}, 0,20-0,40 \mathrm{~m}$ e $0,40-0,60 \mathrm{~m}$ de profundidade. 
As colunas foram montadas considerando-se o perfil do solo retirado e as quantidades isotônicas de solo em todas as colunas ( $3 \mathrm{~kg}$ de solo por coluna).

As colunas eram constituídas de aço inox com $70 \mathrm{~mm}$ de diâmetro, permitindo acondicionar $50 \mathrm{~cm}^{3}$ de solo. A extremidade inferior de cada coluna foi revestida com tela de 100 mesh, para evitar perda de solo ou turvamento das amostras do material que seria coletado.

$\mathrm{Na}$ Tabela 2, são apresentadas algumas características físicas e hidrodinâmicas dos solos coletados dos tratamentos e condicionados nas colunas de solo deformado e indeformado.

TABELA 2. Características físicas e hidrodinâmicas dos solos dos respectivos tratamentos nas colunas de solo deformado e indeformado. Physical and hydrodynamic characteristics of soil from the treatments in deformed and undeformed soil columns.

\begin{tabular}{ccccccccccc}
\hline \multirow{2}{*}{ Parâmetro } & \multirow{2}{*}{ Unidade } & \multicolumn{4}{c}{ Colunas de Solo Deformado } & \multicolumn{3}{c}{ Colunas Solo Indeformado } \\
\cline { 2 - 10 } & $\mathrm{T} 1$ & $\mathrm{~T} 2$ & $\mathrm{~T} 3$ & $\mathrm{~T} 4$ & $\mathrm{~T} 1$ & $\mathrm{~T} 2$ & $\mathrm{~T} 3$ & $\mathrm{~T} 4$ \\
\hline $\begin{array}{c}\text { Massa total de } \\
\text { solo na coluna }\end{array}$ & $\mathrm{kg}$ & 3,00 & 3,00 & 3,00 & 3,00 & 1,96 & 1,63 & 1,63 & 1,63 \\
\hline $\begin{array}{c}\text { Volume total de } \\
\text { solo na coluna }\end{array}$ & $\mathrm{L}$ & 2,40 & 2,18 & 1,98 & 1,87 & 2,04 & 2,00 & 2,12 & 1,98 \\
\hline Porosidade & $\%$ & 64,00 & 68,00 & 66,00 & 67,00 & 64,00 & 68,00 & 66,00 & 67,00 \\
\hline Umidade Inicial & $\mathrm{cm}^{3} \mathrm{~cm}^{-3}$ & 0,26 & 0,23 & 0,25 & 0,28 & 0,25 & 0,10 & 0,16 & 0,18 \\
\hline $\begin{array}{c}\text { Massa específica } \\
\text { do solo na coluna }\end{array}$ & $\mathrm{g} \mathrm{g}^{-1}$ & 1,00 & 1,10 & 1,21 & 1,28 & 0,96 & 0,82 & 0,77 & 0,82 \\
\hline $\begin{array}{c}\text { Massa específica } \\
\text { do solo in situ }\end{array}$ & $\mathrm{g} \mathrm{g}^{-1}$ & 1,01 & 0,95 & 0,95 & 1,02 & 1,01 & 0,95 & 0,95 & 1,02 \\
\hline $\begin{array}{c}\text { Tempo para o } \\
\text { início do } \\
\text { escoamento }\end{array}$ & $\mathrm{min}$ & 39,52 & 49,55 & 53,43 & 65,23 & 35,20 & 4,00 & 300,00 & 42,00 \\
\hline
\end{tabular}

Depois de as colunas serem fixadas verticalmente em uma estrutura de madeira, foram submetidas à aplicação de água destilada, utilizando-se de permeâmetro de carga constante, com $6 \mathrm{~cm}$ de carga hidráulica, alimentado por uma garrafa de Mariotte. A quantidade total de água destilada aplicada em cada coluna correspondeu acerca de 4,0 volumes de poros. Foram coletados $100 \mathrm{~mL}$ por amostra de percolado, correspondente a aproximadamente 45 amostras de percolado por coluna. Optou-se por determinar quatro a cinco pontos para a construção da curva, visando à transformação linear, haja vista que esses pontos são suficientes para a determinação da reta.

Nas amostras percoladas, determinaram-se os parâmetros nitrato $\left(\mathrm{NO}_{3}\right)$, potássio $(\mathrm{K})$ (APHA, AWWA, WEF, 1998) e a condutividade elétrica, usando condutivímetro de bancada.

A dinâmica da lixiviação dos íons nas colunas de solo deformado e indeformado, para os quatro tratamentos considerados, foi analisada a partir da construção de curvas de concentração relativa $\left(\mathrm{C} / \mathrm{C}_{0}\right)$ versus o tempo ou o número de volume de poros nos efluentes recolhidos (BEHERA et al., 2003).

Após a obtenção das curvas de distribuição em cada tratamento, para cada parâmetro, com amostras de solo deformado e indeformado, foi realizado o teste de comparação entre as duas curvas com a finalidade de verificar se as mesmas apresentavam o mesmo comportamento. Como as curvas eram exponenciais, fez-se a transformação ln no eixo $\mathrm{x}$, visando a aplicar análises de regressão e testes estatísticos ("T de student") entre os respectivos coeficientes angular e linear, de modo a verificar diferenças entre as curvas das amostras de solo deformado e indeformado. 


\section{RESULTADOS E DISCUSSÃO}

Observa-se, na Figura 1, que praticamente todo o nitrato, em todos os tratamentos e para as duas metodologias utilizadas (colunas de solo deformado e indeformado), foi lixiviado em 1,0 volume de poros. Resultados semelhantes foram observados por GOMES (2002), utilizando colunas de solo deformado. Entretanto, o processo de lixiviação somente cessou a partir de 3,0 volumes de poros para os tratamentos T2, T3 e T4, nas colunas de solo deformado, e T1, nas colunas de solo indeformado.
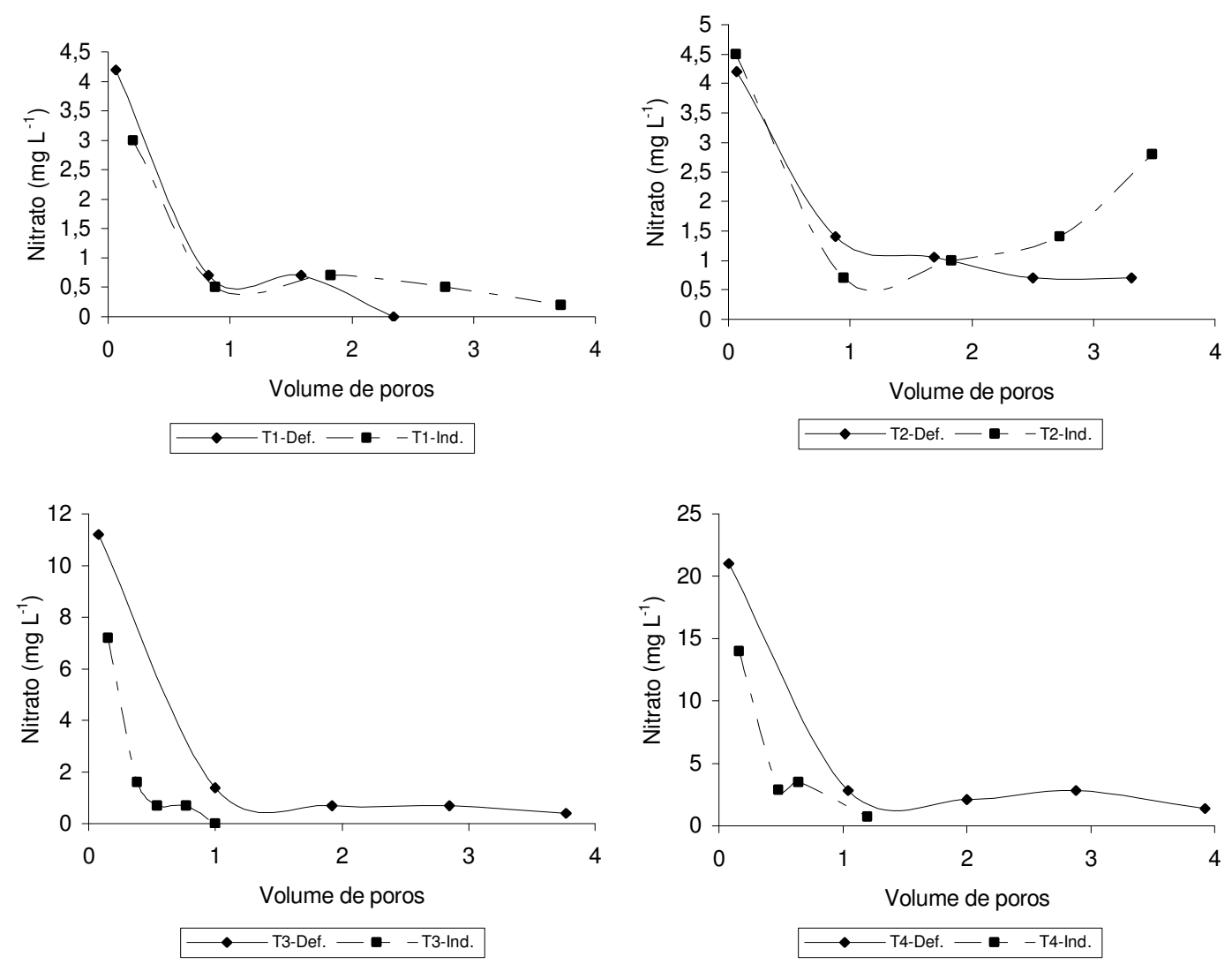

FIGURA 1. Dinâmica de lixiviação do nitrato nas colunas de solo deformado e indeformado para os tratamentos T1, T2, T3 e T4. Dynamics of nitrate leaching in deformed and undeformed soil columns for the treatments T1, T2, T3 and T4.

Na Tabela 3, verificam-se os resultados obtidos por meio do teste de comparação das retas para os valores de nitrato lixiviados.

Observa-se, pela Tabela 3, que as amostras deformadas e indeformadas em cada tratamento obtiveram igualdade de variâncias pelo teste $F\left(\sigma_{D}^{2}=\sigma_{I}^{2}\right)$ e T1, T3 e T4 apresentaram coeficientes angulares iguais pelo teste $\mathrm{T}$, indicando que as duas retas são paralelas. Porém, foram diferentes quanto ao teste $\mathrm{T}$ para coeficiente linear, exceto para o T3. Dessa forma, as retas possuem interceptos diferentes, ou seja, não são coincidentes. Já para T2, nota-se que os resultados apresentaram igualdade, ou seja, as duas retas apresentaram interceptos e inclinações iguais. 
TABELA 3. Teste de comparação dos coeficientes angulares e lineares das curvas das amostras de solo deformado e indeformado para o nitrato lixiviado nos respectivos tratamentos.

Test comparison of angular and linear coefficients of the curves from the samples of deformed and undeformed soil for leachate nitrate in their treatments.

\begin{tabular}{|c|c|c|c|c|c|c|c|c|}
\hline $\mathrm{T}$ & & $\begin{array}{l}\text { Coeficiente } \\
\text { Angular (b) }\end{array}$ & $\begin{array}{c}\text { Coeficiente } \\
\text { Linear (a) }\end{array}$ & QME & $\begin{array}{c}\text { Teste F } \\
\text { QME }\end{array}$ & \multicolumn{2}{|c|}{$\begin{array}{l}\text { Teste T } \\
\text { (b) }\end{array}$} & $\begin{array}{c}\text { Teste T } \\
\text { (a) }\end{array}$ \\
\hline \multirow{2}{*}{$\mathrm{T} 1$} & $\mathrm{D}$ & $-1,15$ & 0,92 & 0,153 & $0,52_{\mathrm{Fc}} \mid<9,55_{\mathrm{Ft}}$ & $1,00_{\mathrm{Tc}}$ & $<2,57_{\mathrm{Tt}}$ & $3,55_{\mathrm{Tc}} \mid>2,45_{\mathrm{Tt}}$ \\
\hline & I & $-0,89$ & 1,20 & 0,293 & $\sigma_{\mathrm{D}}^{2}=\sigma_{\mathrm{I}}^{2} \mathrm{~ns}$ & \multicolumn{2}{|c|}{$\mathrm{b}_{\mathrm{D}}=\mathrm{b}_{\mathrm{I}} \mathrm{ns}$} & $a_{D} \neq a_{I} *$ \\
\hline \multirow{2}{*}{$\mathrm{T} 2$} & $\mathrm{D}$ & $-0,93$ & 1,58 & 0,053 & \begin{tabular}{l|l}
$0,03_{\mathrm{Fc}}$ & $<9,28_{\mathrm{Ft}}$
\end{tabular} & $0,86_{\mathrm{Tc}}$ & $<2,45_{\mathrm{Tt}}$ & \begin{tabular}{l|l}
$1,73_{\mathrm{Tc}}$ & $<2,36_{\mathrm{Tt}}$
\end{tabular} \\
\hline & I & $-0,66$ & 2,09 & 1,734 & $\sigma_{\mathrm{D}}^{2}=\sigma_{\mathrm{I}}^{2} \mathrm{~ns}$ & \multicolumn{2}{|c|}{$\mathrm{b}_{\mathrm{D}}=\mathrm{b}_{\mathrm{I}} \mathrm{ns}$} & $\mathrm{a}_{\mathrm{D}}=\mathrm{a}_{\mathrm{I}} \mathrm{ns}$ \\
\hline \multirow{2}{*}{$\mathrm{T} 3$} & $\mathrm{D}$ & $-2,89$ & 3,15 & 1,762 & \begin{tabular}{l|l}
$1,01_{\mathrm{Fc}}$ & $<9,28_{\mathrm{Ft}}$
\end{tabular} & $3,92_{\mathrm{Tc}}$ & $>2,45_{\mathrm{Tt}}$ & \begin{tabular}{l|l|l}
$6,76_{\mathrm{Tc}}$ & $>2,36_{\mathrm{Tt}}$
\end{tabular} \\
\hline & $\mathrm{I}$ & $-3,80$ & $-0,78$ & 1,734 & $\sigma_{D}^{2}=\sigma_{I}^{2} n s$ & \multicolumn{2}{|c|}{$\mathrm{b}_{\mathrm{D}} \neq \mathrm{b}_{\mathrm{I}} *$} & $a_{D} \neq a_{I} *$ \\
\hline \multirow{2}{*}{$\mathrm{T} 4$} & $\mathrm{D}$ & $-0,75$ & 2,87 & 0,392 & $0,07_{\mathrm{Fc}} \mid<19,00_{\mathrm{Ft}}$ & $0,233_{\mathrm{Tc}}$ & $<2,78_{\mathrm{Tt}}$ & \begin{tabular}{l|l}
$4,97 \mathrm{Tc}$ & $>2,36_{\mathrm{Tt}}$
\end{tabular} \\
\hline & I & $-6,72$ & 0,52 & 4,923 & $\sigma_{D}^{2}=\sigma_{I}^{2} \mathrm{~ns}$ & \multicolumn{2}{|c|}{$\mathrm{b}_{\mathrm{D}}=\mathrm{b}_{\mathrm{I}} \mathrm{ns}$} & $a_{D} \neq a_{I} *$ \\
\hline
\end{tabular}

$\overline{\mathrm{T}}$ - Tratamentos; D - amostras deformadas; I - amostras indeformadas; QME - quadrado médio residual; a - coeficiente angular; $b$ - coeficiente linear; $F_{c}$ - valor de $F$ calculado; $F_{t}$ - ponto crítico da tabela F-Snedecor; $T_{c}$ - valor de $T$ calculado; $\mathrm{T}_{\mathrm{T}}$ - valor crítico da tabela t-Student bicaudal; ns - diferença não significativa a $5 \%$ de probabilidade; * diferença significativa a $5 \%$ de probabilidade.

De acordo com FEIGIN et al. (1991), o nitrato é facilmente lixiviado e aproximadamente 2,0 volumes de poros de água são suficientes para sua completa remoção do solo, sendo tendência observada apenas no comportamento da coluna deformada. Também ao observar a Figura 1, percebe-se que o comportamento dos dados da coluna deformada indicou coerência com o processo físico de lixiviação, ou seja, mostrou-se contínuo e decaiu mais uniformemente ao longo do teste.

Os dados da coluna indeformada, ao contrário, não seguem a tendência natural desse processo físico, com pontos que extrapolam a continuidade do processo. Fato esse comprovado pelo teste de comparação das retas, indicando que o comportamento de lixiviação do nitrato apresentou diferenças nas metodologias de amostra do solo deformado e indeformado. Esses resultados vão ao encontro dos estudos de ROSSI et al. (2007), que também observaram diferenças no transporte do íon nitrato em colunas de solo deformado e indeformado. Entretanto, os mesmos autores relataram que o preenchimento da coluna com amostra de solo deformado não foi representativo para o parâmetro de transporte do íon nitrato, considerando-se, assim, como situação ideal o solo estruturado.

Analisando-se as concentrações iniciais de nitrato nas águas residuárias (Tabela 1) e sua dinâmica de lixiviação nos tratamentos, percebe-se que esse não foi totalmente aproveitado pela cultura da soja. Portanto, devido a sua facilidade de deslocamento para maiores profundidades no solo (VITTI et al., 1994), podendo atingir lençóis freáticos, o nitrato apresenta-se como potencial poluente de águas subterrâneas. Embora o fósforo não seja o objetivo do trabalho, verificaram-se elevadas concentrações desse parâmetro nos diferentes tratamentos aplicados com águas residuárias, o que poderia provocar eutrofização das águas superficiais (CERETTA et al., 2003).

A lixiviação do potássio sofreu estabilização após 1,0 volume de poros nas colunas de solo deformado nos tratamentos T1, T2 e T3, e após 3,0 volumes de poros no tratamento T4 (Figura 2). Nas colunas de solo indeformado, houve estabilidade da concentração de K após aproximadamente 1,$0 ; 0,4$ e 0,5 volume de poros nos tratamentos $\mathrm{T} 1, \mathrm{~T} 3$ e $\mathrm{T} 4$, respectivamente. 

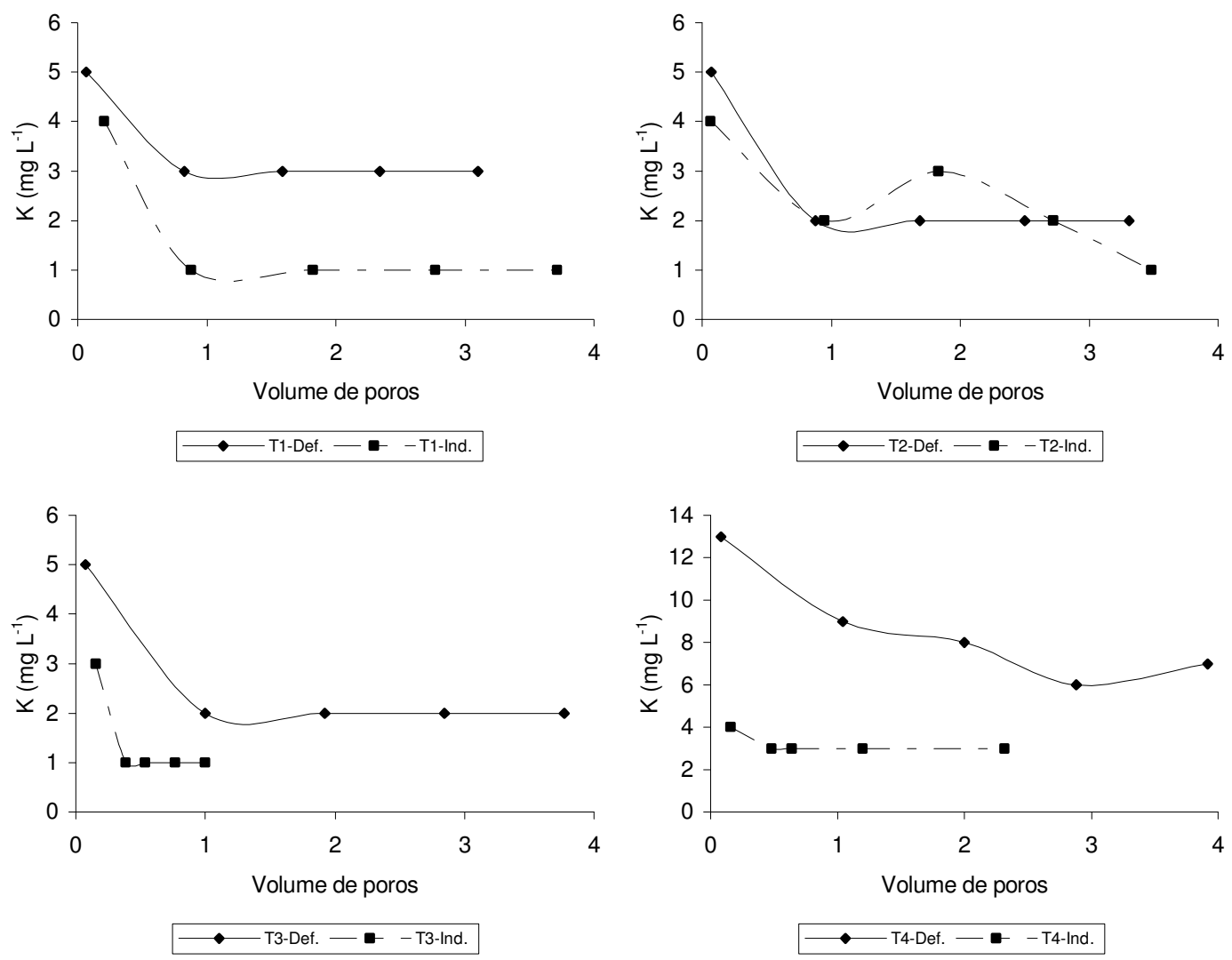

FIGURA 2. Dinâmica de lixiviação do potássio nas colunas de solo deformado e indeformado para os tratamentos T1, T2, T3 e T4. Dynamics of potassium leaching in deformed and undeformed soil columns for the treatments T1, T2, T3 and T4.

$\mathrm{Na}$ Tabela 4, verificam-se os resultados obtidos por meio do teste de comparação das retas para os valores de potássio lixiviados.

TABELA 4. Resultados obtidos por meio do teste de comparação das retas dos valores de potássio lixiviado das amostras deformadas e indeformadas. Results obtained through the comparison test of the arrow values of potassium leachate of the deformed and undeformed samples.

\begin{tabular}{|c|c|c|c|c|c|c|c|c|}
\hline $\mathrm{T}$ & & $\begin{array}{l}\text { Coeficiente } \\
\text { Angular (b) }\end{array}$ & $\begin{array}{l}\text { Coeficiente } \\
\text { Linear (a) }\end{array}$ & QME & $\begin{array}{c}\text { Teste F } \\
\text { QME }\end{array}$ & \multicolumn{2}{|c|}{$\begin{array}{c}\text { Teste T } \\
\text { (b) }\end{array}$} & $\begin{array}{c}\text { Teste T } \\
\text { (a) }\end{array}$ \\
\hline \multirow{2}{*}{$\mathrm{T} 1$} & $\mathrm{D}$ & $-0,54$ & 3,34 & 0,104 & \begin{tabular}{|l|l|}
$0,20_{\mathrm{Fc}}$ & $<9,28_{\mathrm{Ft}}$
\end{tabular} & $4,96_{\mathrm{Tc}}$ & $>2,45_{\mathrm{Tt}}$ & 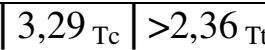 \\
\hline & I & $-1,02$ & 1,85 & 0,522 & $\sigma_{D}^{2}=\sigma_{I}^{2} n s$ & \multicolumn{2}{|c|}{$\mathrm{b}_{\mathrm{D}} \neq \mathrm{b}_{\mathrm{I}} *$} & $a_{D} \neq a_{I} *$ \\
\hline \multirow{2}{*}{$\mathrm{T} 2$} & $\mathrm{D}$ & $-0,81$ & 2,57 & 0,242 & \begin{tabular}{l|l}
$0,46_{\mathrm{Fc}}$ & $<9,28_{\mathrm{Ft}}$
\end{tabular} & $0,58_{\mathrm{Tc}}$ & $<2,45_{\mathrm{Tt}}$ & \begin{tabular}{l|l}
$2,30_{\mathrm{Tc}}$ & $<2,36_{\mathrm{Tt}}$
\end{tabular} \\
\hline & I & $-0,58$ & 2,41 & 0,527 & $\sigma_{D}^{2}=\sigma_{I}^{2} \mathrm{~ns}$ & \multicolumn{2}{|c|}{$\mathrm{b}_{\mathrm{D}}=\mathrm{b}_{\mathrm{I}} \mathrm{ns}$} & $a_{D}=a_{I} n s$ \\
\hline \multirow{2}{*}{$\mathrm{T} 3$} & $\mathrm{D}$ & $-0,81$ & 2,67 & 0,242 & \begin{tabular}{l|l|l}
$0,92_{\mathrm{Fc}}$ & $<9,28_{\mathrm{Ft}}$
\end{tabular} & $5,41_{\mathrm{Tc}}$ & $>2,45_{\mathrm{Tt}}$ & \begin{tabular}{l|l|}
$2,59_{\mathrm{Tc}}$ & $>2,36_{\mathrm{Tt}}$
\end{tabular} \\
\hline & $\mathrm{I}$ & $-1,00$ & 0,60 & 0,262 & $\sigma_{\mathrm{D}}^{2}=\sigma_{\mathrm{I}}^{2} \mathrm{~ns}$ & \multicolumn{2}{|c|}{$\mathrm{b}_{\mathrm{D}} \neq \mathrm{b}_{\mathrm{I}} *$} & $\mathrm{a}_{\mathrm{D}} \neq \mathrm{a}_{\mathrm{I}} *$ \\
\hline \multirow{2}{*}{$\mathrm{T} 4$} & $\mathrm{D}$ & $-1,68$ & 8,81 & 0,499 & \begin{tabular}{l|l}
$0,92_{\mathrm{Fc}}$ & $<9,28_{\mathrm{Ft}}$
\end{tabular} & $17,41_{\mathrm{Tc}}$ & $>2,45 \mathrm{Tt}$ & \begin{tabular}{|l|l|}
$2,56_{\mathrm{Tc}}$ & $>2,36_{\mathrm{Tt}}$
\end{tabular} \\
\hline & I & $-0,35$ & 3,06 & 0,097 & $\sigma_{D}^{2}=\sigma_{I}^{2} n s$ & \multicolumn{2}{|c|}{$b_{D} \neq b_{I} *$} & $a_{D} \neq a_{I} *$ \\
\hline
\end{tabular}

T - Tratamentos; D - amostras deformadas; I - amostras indeformadas; QME - quadrado médio residual; a - coeficiente angular; $\mathrm{b}$ - coeficiente linear; $\mathrm{F}_{\mathrm{c}}$ - valor de $\mathrm{F}$ calculado; $\mathrm{F}_{\mathrm{t}}$ - ponto crítico da tabela F-Snedecor; $\mathrm{T}_{\mathrm{c}}$ - valor de $\mathrm{T}$ calculado; $\mathrm{T}_{\mathrm{T}}$ - valor crítico da tabela t-Student bicaudal; ns - diferença não significativa a $5 \%$ de probabilidade; * diferença significativa a $5 \%$ de probabilidade. 
Verifica-se que tanto as amostras deformadas como as indeformadas para os tratamentos obtiveram igualdade de variâncias pelo teste $F\left(\sigma_{D}^{2}=\sigma_{I}^{2}\right)$. Os tratamentos $T_{1}, T_{3}$ e $T_{4}$ apresentaram coeficientes angulares diferentes pelo teste $\mathrm{T}$ do coeficiente angular, indicando que as duas retas não são paralelas e também foram diferentes quanto ao teste $\mathrm{T}$ para o coeficiente linear. Dessa forma, as retas possuem interceptos diferentes, ou seja, não são coincidentes. Entretanto, para T2, nota-se que os resultados apresentaram igualdade, ou seja, as duas retas apresentam interceptos e inclinações iguais.

As inclinações médias obtidas para todas as curvas de lixiviação do potássio são menores que as inclinações médias do nitrato, indicando menor mobilidade do potássio, devido às cargas negativas que retêm esse elemento presente no solo utilizado (ALCARDE et al., 2000). Essa maior capacidade adsortiva já era esperada, uma vez que o solo das colunas contém maiores teores de argila e de matéria orgânica, resultando em menor avanço do potássio no solo. Resultados semelhantes foram encontrados por ALCÂNTARA \& CAMARGO (2001) para um Latossolo Vermelho eutroférrico do Estado de São Paulo.

Pode-se, ainda, constatar, por meio do teste de comparação das colunas de solo deformado e indeformado, que existiram diferenças no comportamento do potássio lixiviado, ou seja, houve instabilidade no cátion lixiviado para as duas metodologias.

A lixiviação de sais, representada pela condutividade elétrica (Figura 3), sofreu redução com o aumento do volume de poros em todos os tratamentos e para ambas as metodologias de amostragem de solo (deformado ou indeformado).
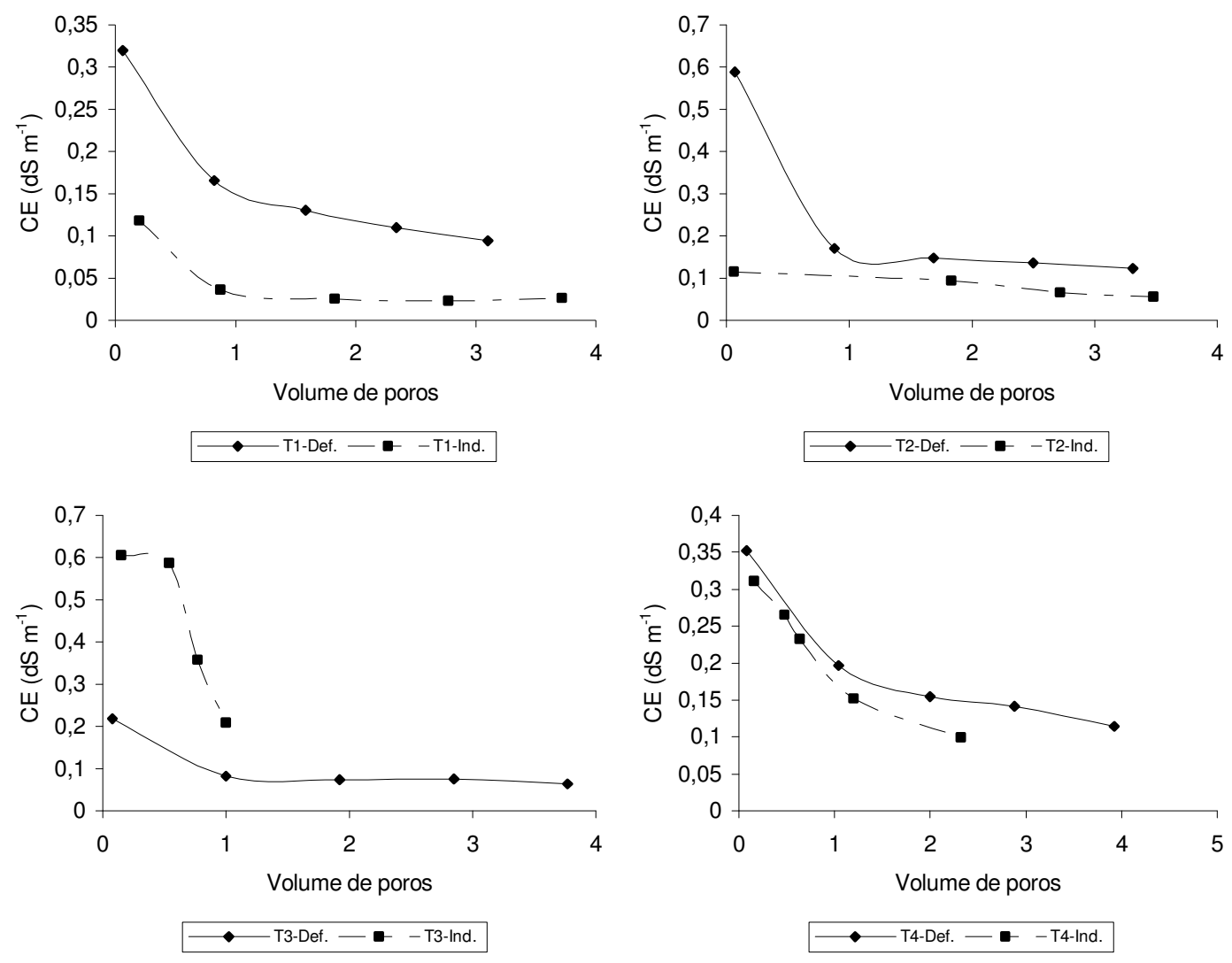

FIGURA 3. Dinâmica da lixiviação de sais (condutividade elétrica) nas colunas de solo deformado e indeformado para os tratamentos T1, T2, T3 e T4. Dynamics of the leaching of salts (electrical conductivity) in columns of deformed and undeformed soil for treatments T1, T2, T3 and T4. 
Apesar da fácil mobilidade dos sais, observou-se que sua concentração inicial lixiviada foi menor que a concentração da água residuária utilizada (Tabela 2).

De modo geral, o tratamento T4, que utilizou maior concentração de ARS na água de irrigação, apresentou, nos lixiviados, os maiores níveis de concentração de nitrato, potássio e concentração de sais, uma vez que apresentam alta solubilidade e, consequentemente, maior mobilidade, considerando-se as colunas de solo deformado.

Na Tabela 5, verificam-se os resultados obtidos por meio do teste de comparação das retas, para os valores de condutividade elétrica nos lixiviados.

TABELA 5. Resultados obtidos através do teste de comparação das retas para os valores de condutividade elétrica no lixiviado das amostras deformadas e indeformadas. Results obtained through the comparison test of the arrows for the values of electrical conductivity of deformed and undeformed leachate samples.

\begin{tabular}{|c|c|c|c|c|c|c|c|c|c|}
\hline $\mathrm{T}$ & & $\begin{array}{l}\text { Coeficiente } \\
\text { Angular (b) }\end{array}$ & $\begin{array}{l}\text { Coeficiente } \\
\text { Linear (a) }\end{array}$ & QME & $\begin{array}{l}\text { Teste F } \\
\text { QME }\end{array}$ & \multicolumn{2}{|c|}{$\begin{array}{l}\text { Teste T } \\
\text { (b) }\end{array}$} & \multicolumn{2}{|c|}{$\begin{array}{l}\text { Teste } \mathrm{T} \\
\text { (a) }\end{array}$} \\
\hline \multirow{2}{*}{$\mathrm{T} 1$} & $\mathrm{D}$ & $-0,058$ & 0,158 & 0,00008 & \begin{tabular}{l|l|}
$0,24_{\mathrm{Fc}}$ & $<9,28_{\mathrm{Ft}}$
\end{tabular} & $0,01_{\mathrm{Tc}}$ & $<2,45_{\mathrm{Tt}}$ & $0,06_{\mathrm{Tc}}$ & $<2,36_{\mathrm{Tt}}$ \\
\hline & I & $-0,032$ & 0,053 & 0,00032 & $\sigma_{D}^{2}=\sigma_{I}^{2} n s$ & \multicolumn{2}{|c|}{$\mathrm{b}_{\mathrm{D}}=\mathrm{b}_{\mathrm{I}} \mathrm{ns}$} & \multicolumn{2}{|c|}{$a_{D}=a_{I} n s$} \\
\hline \multirow[b]{2}{*}{$\mathrm{T} 2$} & D & $-0,124$ & 0,228 & 0,00293 & \begin{tabular}{|l|l}
$12,67_{\mathrm{Fc}}$ & $<19,16_{\mathrm{F}}$
\end{tabular} & $0,10_{\mathrm{Tc}}$ & $<2,36_{\mathrm{Tt}}$ & $0,13_{\mathrm{Tc}}$ & $<2,45_{\mathrm{Tt}}$ \\
\hline & $\mathrm{I}$ & $-0,012$ & 0,082 & 0,00023 & $\sigma_{D}^{2}=\sigma_{I}^{t} n s$ & \multicolumn{2}{|c|}{$\mathrm{b}_{\mathrm{D}}=\mathrm{b}_{\mathrm{I}} \mathrm{ns}$} & \multicolumn{2}{|c|}{$a_{D}=a_{I} n s$} \\
\hline \multirow{2}{*}{$\mathrm{T} 3$} & $\mathrm{D}$ & $-0,040$ & 0,106 & 0,0 & $0,28_{\mathrm{Fc}} \mid<19,16_{\mathrm{Ft}}$ & $0,03_{\mathrm{Tc}}$ & $<2,36_{\mathrm{Tt}}$ & $0,12_{\mathrm{Tc}}$ & $<2,45_{\mathrm{Tt}}$ \\
\hline & I & $-0,184$ & 0,313 & 0,01978 & $\sigma_{D}^{2}=\sigma_{I}^{2} n s$ & \multicolumn{2}{|c|}{$\mathrm{b}_{\mathrm{D}}=\mathrm{b}_{\mathrm{I}} \mathrm{ns}$} & \multicolumn{2}{|c|}{$\mathrm{a}_{\mathrm{D}}=\mathrm{a}_{\mathrm{I}} \mathrm{ns}$} \\
\hline \multirow{2}{*}{$\mathrm{T} 4$} & $\mathrm{D}$ & $-0,060$ & 0,199 & 0,00002 & $0,03_{\mathrm{Fc}} \mid<9,28_{\mathrm{Ft}}$ & $0,03_{\mathrm{Tc}}$ & $<2,45_{\mathrm{Tt}}$ & $0,07_{\mathrm{Tc}}$ & $<2,36_{\mathrm{Tt}}$ \\
\hline & I & $-0,082$ & 0,179 & 0,00052 & $\sigma_{\mathrm{D}}^{2}=\sigma_{\mathrm{I}}^{2} \mathrm{~ns}$ & \multicolumn{2}{|c|}{$\mathrm{b}_{\mathrm{D}}=\mathrm{b}_{\mathrm{I}} \mathrm{ns}$} & \multicolumn{2}{|c|}{$\mathrm{a}_{\mathrm{D}}=\mathrm{a}_{\mathrm{I}} \mathrm{ns}$} \\
\hline
\end{tabular}

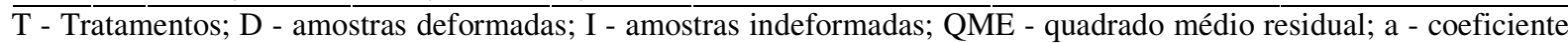
angular; $b$ - coeficiente linear; $F_{c}$ - valor de $F$ calculado; $F_{t}$ - ponto crítico da tabela F-Snedecor; $T_{c}$ - valor de $T$ calculado; $\mathrm{T}_{\mathrm{T}}$ - valor crítico da tabela t-Student bicaudal; ns - diferença não significativa a $5 \%$ de probabilidade; * diferença significativa a $5 \%$ de probabilidade.

Verifica-se que as variâncias das amostras deformadas e indeformadas de todos os tratamentos foram iguais pelo teste $F\left(\sigma_{D}^{2}=\sigma_{I}^{2}\right)$. Observa-se, ainda, que T1, T2, T3 e T4 apresentaram coeficientes angulares iguais, indicando que as duas retas são paralelas pelo teste $\mathrm{T}$ do coeficiente angular e também foram iguais quanto ao teste $\mathrm{T}$ para coeficiente linear. Dessa forma, as retas possuem interceptos iguais, ou seja, são coincidentes.

Inicialmente, notam-se, a partir das Figuras 1; 2 e 3, relativas diferenças entre as colunas de solo deformado ou indeformado, principalmente em relação às concentrações iniciais de nitrato, potássio e condutividade elétrica em um mesmo tratamento. No entanto, as análises estatísticas (Tabelas 3; 4 e 5) indicaram que apenas a condutividade elétrica apresentou o mesmo comportamento para as duas metodologias aplicadas, embora, ao observar a Figura 3, nota-se tendência para o comportamento diferenciado entre as metodologias das amostras deformadas e indeformadas.

Teoricamente, as colunas de solo indeformado caracterizam melhor as condições reais de campo, buscando interferir o mínimo possível na estrutura do solo. Entretanto, durante a amostragem, pode ocorrer compactação do solo e, ainda, caso não seja realizado o perfeito isolamento entre a parede interna da coluna e o solo, pode ocorrer o aparecimento de caminhos preferenciais, interferindo nos resultados obtidos (BERTOL et al., 2005). Possivelmente, isso ocorreu na condução do experimento com as colunas de solo indeformado no tratamento T2. Fato esse também observado nas Tabelas 3 e 4, em que somente T2 apresentou comportamento igual nas amostras de solo deformado e indeformado. 


\section{CONCLUSÕES}

A metodologia de amostragem de colunas de solo indeformado não foi adequada ao estudo da lixiviação de nitrato, potássio e concentração de sais.

Considerando-se os resultados obtidos nas colunas de solo deformado, o nitrato apresentou maior mobilidade no processo de lixiviação, seguido pela concentração de sais e do potássio, proporcionalmente aos níveis de diluição da água residuária de suinocultura nos tratamentos avaliados.

\section{REFERÊNCIAS}

ALCÂNTARA, M.A.K.; CAMARGO, A. Fator de retardamento e coeficiente de dispersão-difusão para o crômio (III) em solos muito intemperizados, influenciados pelo $\mathrm{pH}$, textura e matéria orgânica. Revista Brasileira de Ciência do Solo, Viçosa - MG, v.25, n.1, p.209-216, 2001.

ALCARDE, J.C.; GOMES, P.F.; MALAVOLTA, E. Adubos e adubações. São Paulo: Nobel, 2000. $596 \mathrm{p}$.

ANAMI, M.H.; SAMPAIO, S.C.; SUSZEK, M.; DAMASCENO, S.; QUEIROZ, M.M.F. Deslocamento miscível de nitrato e fosfato proveniente de água residuária da suinocultura em colunas de solo. Revista Brasileira de Engenharia Agrícola e Ambiental, Campina Grande, v.12, n.1, p.75-80, 2008.

ANAMI, M.H.; SAMPAIO, S.C.; SUSZEK, M.; FRIGO, E.P. Lixiviação de nitrato e fosfato proveniente de água residuária da suinocultura tratada em sistema de lagoas. Revista Irriga, Botucatu, v.12, n.2, p.192-201, 2007.

APHA, AWWA, WEF. Standard methods for the examination of water and wastewater. $20^{\text {th }}$ ed. Washington: American Public Health Association, 1998. 1.193 p.

BAUMGARNTER, D.; SAMPAIO, S.C.; SILVA, T.R.; VILAS BOAS, M.A. Reúso de águas residuárias da piscicultura e da suinocultura na irrigação da cultura da alface. Engenharia Agrícola, Jaboticabal, v.27, n.1, p.152-163, 2007.

BEHERA, S.; JHA, K.M.; KAR, S. Dynamics of water flow and fertilizer solute leaching in lateritic soils of Kharagpur region, India. Agricultural Water Management, Kharagpur, v.63, n.1, p.77-98, 2003.

BERTOL, O.J.; RIZZI, N.E.; FAVARETTO, N.; LAVORANTI, O.J. Perdas de nitrogênio via superfície e subsuperfície em sistema de semeadura direta. Floresta, Curitiba, v.35, n.3, p.429-442, 2005.

BREDA, C.A. Desenvolvimento de um penetrômetro de placa de aço e avaliação em campo da compactação do solo. 2003. Dissertação (Mestrado em Engenharia de Sistemas Agroindustriais) Universidade Estadual do Oeste do Paraná, Cascavel, 2003.

CAOVILLA, F.A.; SAMPAIO, S.C.; PEREIRA, J.O.; VILAS BOAS, M.A.; GOMES, B.M. ; FIGUEIRÊDO, A.C. Lixiviação de nutrientes provenientes de águas residuárias em colunas de solo cultivado com soja . Revista Brasileira de Engenharia Agrícola e Ambiental, Campina Grande, v.9, suplemento, p.283-287, 2005.

CERETTA, C.A.; DURIGON, R.; BASSO, J.C.; BARCELLOS, L.A.R.; VIEIRA, F.C.B.

Características químicas de solo sob aplicação de esterco líquido de suínos em pastagem natural. Pesquisa Agropecuária Brasileira, Brasília, v.38, n.6, p.729-735, 2003.

COSTA, S.N.; MARTINEZ, M.A.; MATOS, A.T.; RAMOS, V.B.N. Mobilidade do nitrato em colunas de solo sob condições de escoamento não permanente. Revista Brasileira de Engenharia Agrícola e Ambiental, Campina Grande, v.3, n.2, p.190-194, 1999. 
DAL BOSCO, T.C.; SAMPAIO, S.C.; OPAZO, M.A.U.; GOMES S.D.; NÓBREGA, L.H.P. Aplicação de água residuária de suinocultura em solo cultivado com soja: cobre e zinco no material escoado e no solo. Engenharia Agrícola, Jaboticabal, v.28, n.4, p.699-709, 2008.

EMBRAPA. EMPRESA BRASILEIRA DE PESQUISA AGROPECUÁRIA. Centro Nacional de Pesquisa de Solos. Sistema brasileiro de classificação dos solos. Rio de Janeiro, 1999. 412 p.

EMBRAPA. EMPRESA BRASILEIRA DE PESQUISA AGROPECUÁRIA. Levantamento sistemático da produção e abate de suínos. 2006. Disponível em:

<http://www.cnpsa.embrapa.br/?ids=So6f90o4t>. Acesso em: 24 jul. 2006.

FEIGIN, A.; RAVINA, I.; SHALHEVET, J. Irrigation whit treated sewage effluent, management for environmental protection: advanced series in agricultural sciences. Berlin: Springer-Verlang, 1991. $224 \mathrm{p}$.

FRIGO, E.P.; SAMPAIO, S.C.; FREITAS, P.S.L.; QUEIROZ, M.M.F.; NÓBREGA, L.H.P.; MALLMANN, L.S. Desempenho do sistema de gotejamento e de filtros utilizando água residuária da suinocultura. Revista Irriga, Botucatu, v.11, n.3, p.305-318, 2006.

GOMES, E. Movimento de nitrato proveniente de água residuária de frigorífico em colunas de solo. 2002. Dissertação (Mestrado em Engenharia Agrícola) - Universidade Estadual do Oeste do Paraná, Cascavel, 2002.

GOMES, E.R.S.; SAMPAIO, S.C.; CORRÊA, M.M.; VILAS BOAS, M.A.; ALVES, L.F.A; ALVES SOBRINHO, T. Movimento de nitrato proveniente de água residuária em colunas de solo. Engenharia Agrícola, Jaboticabal, v.24, n.3, p.557-568, 2004.

KONRAD, M. Efeito de sistemas de irrigação localizada sobre a produção e qualidade da acerola (Malpighia spp) na região da Nova Alta Paulista. 2002. 134 f. Dissertação (Mestrado em Sistema de Produção) Faculdade de Engenharia, Universidade Estadual Paulista, Ilha Solteira, 2002.

LONGO, A.J.; SAMPAIO, S.C.; SUSZEK, M. Equação de chuvas intensas e precipitação provável para o município de Cascavel - PR. Varia Scientia, Cascavel, v.6, n.12, p.16-30, 2006.

PELISSARI, R.; SAMPAIO, S.C.; GOMES, S.D.; CREPALLI, M.S. Lodo têxtil e água residuária da suinocultura na produção de mudas de eucalyptus grandis (W, Hill ex Maiden). Engenharia Agrícola, Jaboticabal, v.29, n.2, p.288-300. 2009.

ROSSI, P.; MIRANDA, J.H.; DUARTE, S.N. Curvas de distribuição de efluentes do íon nitrato em amostras de solo deformadas e indeformadas. Engenharia Agrícola, Jaboticabal, v.27, n.3, p.675682, 2007.

SAMPAIO, S.C.; SILVESTRO, M.G.; FRIGO, E.P.; BORGES, C.M. Relação entre série de sólidos e condutividade elétrica em diferentes águas residuárias. Revista Irriga, Botucatu, v.12, n.4, p.557$562,2007$.

SANTOS, A.B.; FAGERIA, N.K.; ZIMMERMANN, F.J.P. Atributos químicos do solo afetado pelo manejo da água e do fertilizante potássico na cultura de arroz irrigado. Revista Brasileira de Engenharia Agrícola e Ambiental, Campina Grande, v.6, n.1, p.12-16, 2002.

SUSZEK, M.; SAMPAIO, S.C.; SANTOS, R.F.; NUNES, O.L.G.S.; DAMASCENO, S.; MALLMANN, L.S. Uso de água residuária da suinocultura na bioestabilização de resíduos verdes urbanos. Revista Brasileira de Engenharia Agrícola e Ambiental, Campina Grande, v.9, n.4, p.176180, 2005.

VITTI, G.C.; BOARETTO, A.E.; PENTEADO, S.R. Fertilizantes e fertirrigação. In: BOAS, R.L.V.; BOARETTO, A.E.; VITTI, G.C. Fertilizantes fluidos. Piracicaba: Potafós, 1994. p.237298. 